\title{
INVESTIGATION OF CHAOTIC-TYPE FEATURES IN HYPERSPECTRAL SATELLITE DATA
}

\author{
Osman Kocal and Mufit Cetin \\ Department of Computer Engineering, Yalova University, Yalova, Turkey \\ osman.kocaleyalova.edu.tr, mufit.cetineyalova.edu.tr
}

\begin{abstract}
Hyperspectral images provide detailed spectral information with more than several hundred channels. On the other hand, the high dimensionality in hyperspectral images also causes to classification problems due to the huge ratio between the number of training samples and the features. In this paper, Lyapunov Exponents (LEs) are used to determine chaotic-type structure of EO- 1 Hyperion hyperspectral image, a mixed forest site in Turkey. Experimental results demonstrate that EO-1 Hyperion image has a chaotic structure by checking distribution of Lyapunov Exponents (LEs) and they can be used as discriminative features to improve classification accuracy for hyperspectral images.
\end{abstract}

\section{KEYWORDS}

Hyperspectral Image, EO-1 Hyperion, Phase Space, Chaos, Lyapunov Exponents

\section{INTRODUCTION}

Hyperspectral sensors collect the electromagnetic spectrum of reflected light from objects and combine spectral information for each pixel in the image of a scene. Every pixel in the image represents to a unique 'fingerprints' of the object, also known as a spectral signature and provides more spectral information to characterize different materials [1]. Therefore, hyperspectral images are much more functional to identify different objects than multispectral images. With the recent advances in sensor technology, hyperspectral images including AVIRIS and EO-1 HYPERION have become available and used in many application areas such as forestry, mining, oil industry, agriculture, ecology, environmental monitoring and geology etc [2, 3, 4, 5].

Hyperspectral images provide detailed spectral information with more than several hundred channels. On the other hand, the high dimensionality in hyperspectral images also causes to classification problems due to the huge ratio (the Hughes phenomenon) between the number of training samples and the features [6,7]. Besides, high dimensionality of hyperspectral images causes the failures in common image classification algorithms and high computational complexity due to hundreds of bands. Therefore, dimension reduction is an important research topic for classification of hyperspectral images on remote sensing.

The purpose of dimension reduction ideally is to preserve as much as possible the information of original hyperspectral data set. Thus, feature extraction is an important way to reduce the

Jan Zizka et al. (Eds) : CCSEIT, MoWiN, IT, AIAP, ICBB - 2015

pp. 49-53, 2015. (C) CS \& IT-CSCP 2015

DOI : $10.5121 / \mathrm{csit} .2015 .51106$ 
dimension of the hyperspectral image without a subspace from the original data like feature selection method $[8,9]$. In this context, the purpose of the study is to reduce the dimension of the hyperspectral data from hundreds of bands to only a few features using Lyapunov Exponents (LEs) of original reflectance curves.

\section{CHAOTIC STRUCTURE ANALYSIS USING LYAPUNOV EXPONENTS}

Phase spaces of spectral signals are crucial to identify relationship between spectral signals and chaotic structure of image. Phase spaces allow observing signal dynamics in a spectral band series and providing understandable important information related to the chaotic dynamics of the original signal. The hyperspectral reflectance curves for each class have similar patterns and they can be used as a time series depend on the number of spectral bands used in the imaging system. The constant spectral band difference between the neighboring elements of each reconstructed vector, which is the embedding dimension of the phase space, is represented by the spectral band for each pixel [10]. The phase-space vector of the spectral signal is reconstructed as follows:

$n=p \cdot \hat{k}+b$

$s[n\rceil=(x\lceil n\rceil, x\lceil n+1\rceil, \ldots x[n+p . k-1])$

Where $\mathrm{n}$ denotes index of data point in 1-D spectral signal, $\mathrm{x}[\mathrm{n}]$ is the nth sample of the spectral signal, $\mathrm{k}$ indicates the total number of spectral bands, $\mathrm{b}$ denotes index of the spectral band for selected pixel on hyperspectral image and $\mathrm{p}$ is number of pixel, as numbers between 0 and total number of samples.

Lyapunov exponents provide a rapid effective method to determine chaotic structure in dynamic systems $[11,12]$. Furthermore, Lyapunov exponents are a quantitative measure of the rate at nearby orbits converge and diverge. In the system, having more than one positive and negative Lyapunov exponents indicate chaotic behaviour [13, 14, 15]. There are many algorithms proposed in the literature for estimating the Lyapunov exponents. The most popular from these algorithms is from Abarbanel et al [16].

$\lambda=\lim _{n \rightarrow \infty} \frac{1}{N} \sum_{n=1}^{N} l n \frac{d(s(n+1), s(m+1))}{d(s(n), s(m))}$

where, $s(n)$ denotes the reference point, $s(m)$ is the nearest neighbour of $s(n)$ on a nearby trajectory, $d(s(n), s(m))$ is the initial distance between the nearest neighbours, $d(s(n+1), s(m+1))$ is the distance between $\mathrm{s}(\mathrm{n}+1)$ and $\mathrm{s}(\mathrm{m}+1)$ which are the next pair of neighbours on their trajectories $[17,18]$.

\section{TEST DATASET}

Earth Observing 1 (EO-1) HYPERION, launched on 21 November 2000 by NASA, is the first hyperspectral satellite. The Hyperion sensor detects a total of 242 channels from 356 to $2577 \mathrm{~nm}$ at approximately a $10-\mathrm{nm}$ spectral resolution with a $30 \mathrm{~m}$ spatial resolution and scans a ground area approximately $7.7 \mathrm{~km}$ in the across-track direction, and $42 \mathrm{~km}$ in the along-track direction. Test image covers a portion of Sundiken Mountains, a mixed forest site, in Eskisehir province, Turkey. In the study, atmospheric absorption bands and low signal-to-noise ratio bands were eliminated from the dataset and remained 153 bands are used for analysis $[19,20]$. In the study area, four object classes including pine and oak trees, grassland and water are selected to evaluate Lyapunov exponents as a feature tool. 


\section{RESULTS}

Lyapunov Exponents (LEs) are used to demonstrate the chaotic-type features for EO-1 Hyperion hyperspectral data set in the study. First, 1-D hyperspectral spectral vectors are created as a chaotic series and combined from manually selected pixels for each class in the image, remaining in 153 healthy channels of Hyperion data (Figure 1).

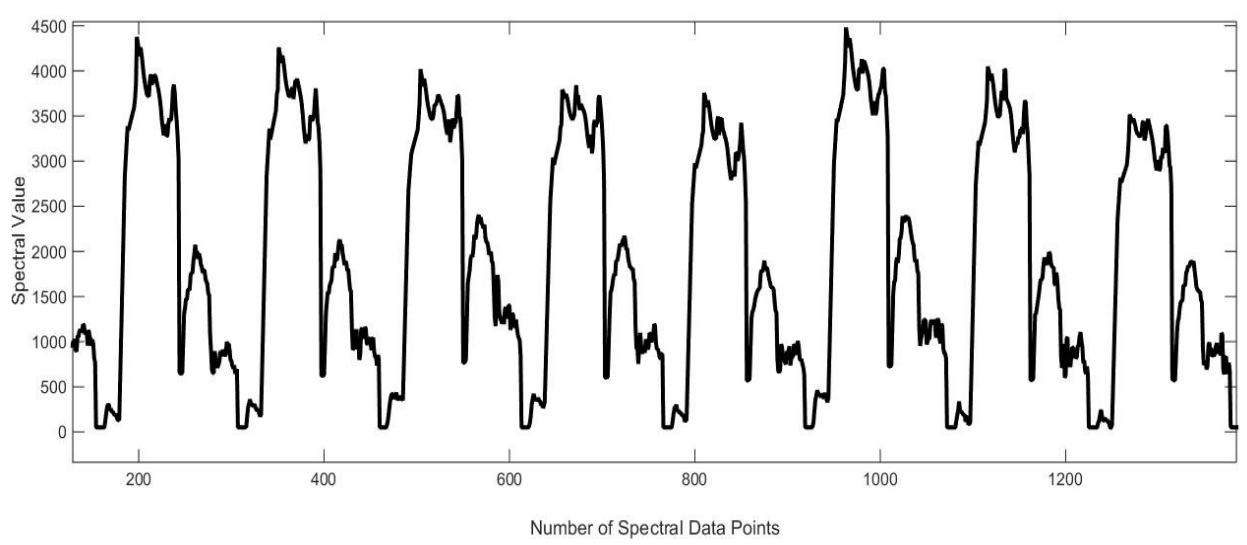

Figure 1. Spectral series of pixels for class Oak trees

Then, phase spaces of spectral signals are initially produced to explore the chaotic structure of hyperspectral image using 1-D spectral vectors (Figure 2). Next, Lyapunov Exponents (LEs) of the spectral signals are calculated for four objects including pine, oak, grassland and water and it is observed different negative or minus signs among them.

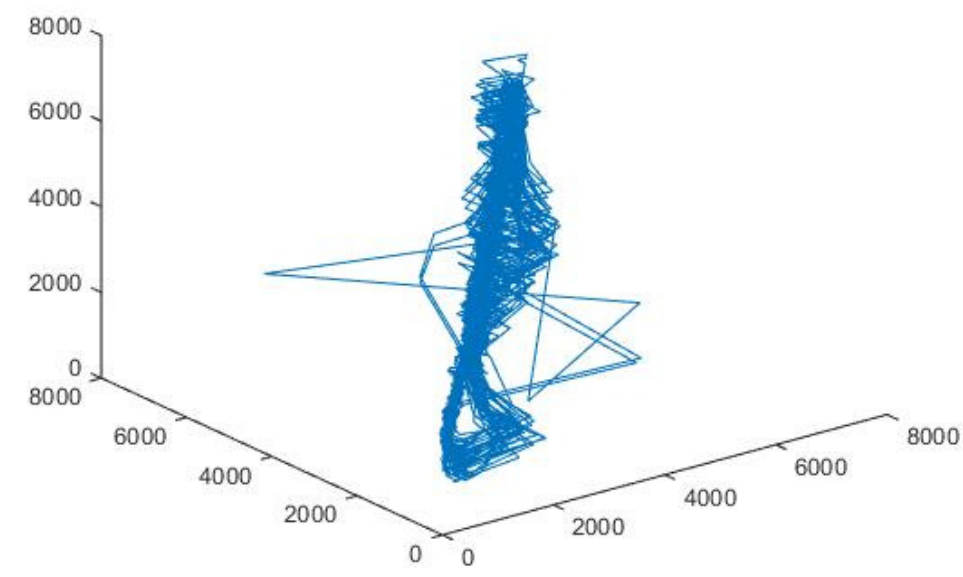

Figure 2. Reconstructed phase space of the spectral signal in 3-D

A positive and negative Lyapunov exponents are computed in all the hyperspectral signals setting total number of data points $(n=3060)$. Positive and negative Lyapunov exponents indicate the possible existence of chaotic behaviour in hyperspectral data (Table1). Kaplan-Yorke dimension (DKY) indicates the fractal dimension of the reconstructed signal corresponding Lyapunov spectrum and values of the embedding dimension $(\mathrm{m})[13,11]$. 
Table 1. The Lyapunov exponents for 4738 trials each with embedding dimension 5 calculated for each object

\begin{tabular}{|c|c|c|c|c|c|c|c|c|c|c|}
\hline \multirow{2}{*}{$\begin{array}{c}\text { Type } \\
\text { of } \\
\text { Object }\end{array}$} & \multirow{2}{*}{$\begin{array}{c}\text { Number } \\
\text { of Iteration }\end{array}$} & \multirow{2}{*}{ m } & \multicolumn{5}{|c|}{ Lyapunov Exponents } & \multirow{2}{*}{$\begin{array}{l}\text { Average Absolute } \\
\text { Forecast Errors }\end{array}$} & \multirow{2}{*}{$\begin{array}{c}\text { Average } \\
\text { Neighborhood } \\
\text { Size }\end{array}$} & \multirow{2}{*}{ DKY } \\
\hline & & & $\mathbf{\Lambda 1}$ & $\Lambda 2$ & $\mathbf{\Lambda 3}$ & $\wedge 4$ & $\Lambda 5$ & & & \\
\hline pine & 4738 & 5 & 0.1632 & -0.0272 & -0.1756 & -0.3697 & -0.8537 & 106 & 117 & 2.77 \\
\hline oak & 4738 & 5 & 0.1851 & -0.0155 & -0.1831 & -0.3611 & -0.8469 & 177 & 185 & 2.93 \\
\hline grassland & 4738 & 5 & 0.1408 & -0.0031 & -0.1791 & -0.3603 & -0.7431 & 207 & 187 & 2.62 \\
\hline water & 4738 & 5 & 0.3241 & 0.0529 & -0.1093 & -0.3118 & -0.7257 & 54 & 39 & 3.86 \\
\hline
\end{tabular}

\section{CONCLUSION}

The experimental results demonstrate that EO-1 Hyperion image has a chaotic structure by checking distribution of Lyapunov Exponents (LEs) and they can be used as discriminative features to improve classification accuracy for hyperspectral images. Future work, therefore, includes the investigation and evaluation of the effects of chaotic features on hyperspectral image classification.

\section{REFERENCES}

[1] Sarath, T. \& Nagalakshmi, G., (2014) "A Land Cover Fuzzy Logic Classification By Maximumlikelihood", International Journal of Computer Trends and Technology, Vol. 13, No.2, pp56-60.

[2] Carter, G.A. \& Miller, R.L., (1994) "Early detection of plant stress by digital imaging within narrow stress-sensitive wavebands", Remote Sensing of Environment, Vol. 50, pp295-302.

[3] Crosta, A.P., Sabine, C. and Taranik, J.V., (1998), "Hydrothermal Alteration Mapping at Bodie, California, Using AVIRIS Hyperspectral Data", Remote Sensing of Environment, Vol.65, No.3,pp 309-319.

[4] Ellis, J.M., Davis, H.H., Zamudio, J.A., (2001), "Exploring for onshore oil seeps with hyperspectral imaging", Oil and Gas Journal, Vol. 99, No. 37, pp49-56.

[5] Horig, B., Kuhn, F., Oschutz, F. and Lehmann, F., (2001), "HyMap hyperspectral remote sensing to detect hydrocarbons", Int. J. Remote Sensing, Vol. 22, No.8, pp1213-1422.

[6] Mianji, F.A. \& Zhang, Y., (2011), "Robust hyperspectral classification using relevance vector machine", IEEE Trans. Geosci. Remote Sens., Vol.49, pp2100-2112.

[7] Zhang, X., He, Y., Zhou, N., and Zheng, Y., (2013), "Semisupervised dimensionality reduction of hyperspectral images via local scaling cut criterion", IEEE Geoscience Remote Sensing Letters, Vol. 10, No.6, pp1547-1551.

[8] Shaw, G. \& Manolakis, D., (2002), "Signal processing for hyperspectral image exploitation", IEEE Signal Process. Mag., Vol. 19, No.1, pp12-16.

[9] Semeniv, O. V., Shatokhina, Y. V., and Yatsenko, V. A., (2008), "Validation of hyperspectral data classification models", J. Autom. Inf. Sci., Vol. 40, No.5, pp46-51.

[10] Jihao, Y., Chao, G. and Xiuping, J, (2012), "Using Hurst and Lyapunov Exponent For Hyperspectral Image Feature Extraction", IEEE Geoscience and Remote Sensing Letters, Vol. 9, No. 49, pp705 709.

[11] Banbrook, M. \& McLughlin, S., (1994), "Is speech chaotic?: Invariant geometrical mesures for speech data", IEEE Colloquiunz on Exploiting Chaos in Signals Processing, Digest No 1994/193, pp8/1-8/10.

[12] Eckmann, J.P. , Kamphorst, O.S., Ruelle, D. and Ciliberto, S., (1986), "Lyapunov exponents from time series", Phys. Rev. A, Vol. 34, pp4971-4979.

[13] Lorenz, E.N., (1963), "Deterministic nonperiodic flow", J. Atmos. Sci. , Vol.20, No.2, pp130-141.

[14] Wolf, A., Swift, J.B., Swinney, H.L. and Vastano, J.A. , (1985), "Determining Lyapunov exponents from a time series", Physica D, Vol. 16, pp285-317. 
[15] Kocal, O.H., Yuruklu, E., Avcibas, E., (2008), "Chaotic-type features for speech steganalysis", IEEE Transactions on Information Forensics and Security, Vol. 3,No.4, pp651-661.

[16] Abarbanel, H.D.I., (1996), Analysis of Observed Chaotic Data, 1st ed. New York: Springer-Verlag.

[17] Kokkinos, I. \& Maragos, P., (2005), "Nonlinear speech analysis using models for chaotic systems", IEEE Trans. Speech Audio Process., Vol. 13, No.6, pp1098-1109.

[18] Martinez, F., Guillamon, A., Alcaraz, J.C., and Alcaraz, M.C., (2002), "Detection of chaotic behavior in speech signals using the largest Lyapunov exponent", in Proc. 14th Int Conf. Digital Signal Processing, Jul. 1-3, 2002, 1, pp317-320.

[19] Parkinson, C.L., Ward, A., King, M.D., and editors, (2006), Earth Science Reference Handbook: A Guide to NASA's Earth Science Program and Earth Observing Satellite Missions. Washington, D.C.: National Aeronautics and Space Administration.

[20] Cetin, M. \& Musaoglu, N., (2009), "Merging hyperspectral and panchromatic image data: qualitative and quantitative analysis", Int. J. Remote Sens., Vol. 30, No.7, pp1779-1804.

\section{AUTHORS}

Osman Hilmi Kocal was born in Istanbul, Turkey, in 1967. He received the B.Sc., M.Sc., and Ph.D. degrees in electronics and telecommunication engineering from the Technical University of Istanbul, Istanbul, in 1989, 1992, and 1998, respectively. He was a Research Assistant with the Technical University of Istanbul and Turkish Air Force Academy, Istanbul, respectively. Currently, he is an Assistant Professor with the Department of Computer Engineering, Yalova University, Yalova, Turkey. His research interests include chaotic signals and adaptive signal processing.

Mufit Cetin is an associate professor in the department of Computer Engineering at Yalova University. He received his BS, MS and PhD degrees in Geomatics Engineering from Yildiz Technical University in 1996, Institute of Gebze Technology in 2001 and Istanbul Technical University in 2007, respectively. His current research interests include image processing and remote sensing. 